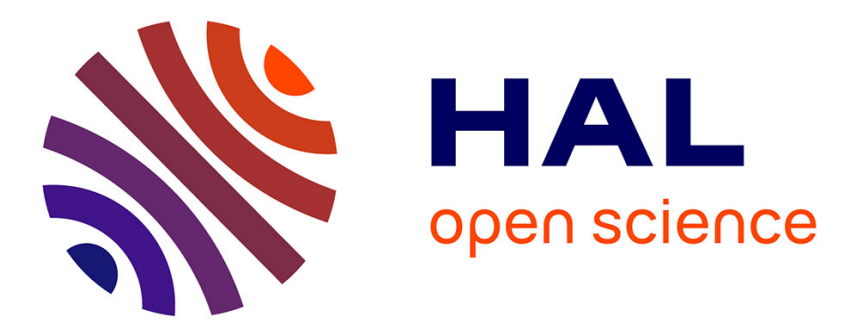

\title{
Improved calibration of simulation models in railway dynamics: application of a parameter identification process to the multi-body model of a TGV train
} Sönke Kraft, Guillaume Puel, Denis Aubry, Christine Fünfschilling

\section{- To cite this version:}

Sönke Kraft, Guillaume Puel, Denis Aubry, Christine Fünfschilling. Improved calibration of simulation models in railway dynamics: application of a parameter identification process to the multi-body model of a TGV train. Vehicle System Dynamics, 2013, 51 (12), pp.1938-1960. 10.1080/00423114.2013.847467 . hal-00921785

\section{HAL Id: hal-00921785}

https://hal-centralesupelec.archives-ouvertes.fr/hal-00921785

Submitted on 21 Dec 2013

HAL is a multi-disciplinary open access archive for the deposit and dissemination of scientific research documents, whether they are published or not. The documents may come from teaching and research institutions in France or abroad, or from public or private research centers.
L'archive ouverte pluridisciplinaire HAL, est destinée au dépôt et à la diffusion de documents scientifiques de niveau recherche, publiés ou non, émanant des établissements d'enseignement et de recherche français ou étrangers, des laboratoires publics ou privés. 


\title{
Improved calibration of simulation models in railway dynamics: application of a parameter identification process to the multi-body model of a TGV train
}

\author{
S. Kraft ${ }^{\mathrm{a} *}$, G. Puel ${ }^{\mathrm{b}}$, D. Aubry ${ }^{\mathrm{b}}$ and C. Funfschilling ${ }^{\mathrm{a}}$ \\ ${ }^{\mathrm{a}} \mathrm{SNCF}$, France; b Laboratoire MSSMat (Ecole Centrale Paris / CNRS UMR8579), \\ Grande Voie de Vignes, Chatenay-Malabry, France
}

(Received 00 Month 200x; final version received 00 Month 200x)

\begin{abstract}
This paper aims at estimating the vehicle suspension parameters of a TGV train from measurement data. A better knowledge of these parameters is required for virtual homologation or condition monitoring applications. The estimation of the parameter values is performed by minimizing a misfit function describing the distance between the measured and the simulated vehicle response. Due to the unsteady excitation from the real track irregularities and nonlinear effects in the vehicle behaviour, the misfit function is defined in the time domain using a least squares estimation. Then an optimization algorithm is applied in order to find the best parameter estimate within the defined constraints. The complexity of the solution surface with many local minima requires the use of global optimization methods. The results show that the model can be improved by this approach providing a response of the simulation model closer to the measurements.
\end{abstract}

Keywords: railway vehicle dynamics, parameter identification, global optimization

\section{Introduction and motivation}

The simulation of the dynamic behaviour of railway vehicles using multi-body programs has been used by constructors for many years. It allows to compare different design solutions virtually thus avoiding prototypes and decreasing development time and costs. These applications are focused on relative differences between several constructive solutions rather than on very precise absolute values of the vehicle response.

With improved models and faster computing times new application fields for simulations of the dynamic response of railway vehicles are explored. These are the virtual homologation of new vehicles, the condition-based maintenance and the definition of track maintenance procedures depending on the vehicle response. The virtual homologation aims at replacing some of the costly inline tests of new or modified vehicles by simulations. The homologation has to guarantee that the vehicle response is within the limits defined in the standard EN14363 [1] for vehicle acceptance.

The condition-based maintenance is based on detailed information about the actual state of vehicle elements. Up to now it is based on signal processing from measured data. Applications are the measurement of axle box accelerations for the detection of wheel defects and the identification of running instability as outlined

\footnotetext{
*Corresponding author. Email: sonke.kraft@sncf.fr
} 
in [2]. Alternatively nominal simulation models can be used to detect property changes in particular vehicle elements. Different methods for model-based condition monitoring are outlined in [3]. In [4] Li discusses the model-based estimation of suspension parameters. In his work a filter-based method is used for parameter identification of a multi-body model.

The track maintenance requires limit values for the admissible amplitude of track defects. These can be obtained by simulating the vehicle response as a function of the track geometry. For all these applications the interest does not lie in relative changes of the vehicle response but in precise absolute values. They require therefore a very accurate modelling of the vehicle response.

The capacity of the model to reproduce the dynamic response of the real vehicle depends on the one hand on the structure of the model and on the other hand on the choice of the model parameters. Only the dynamic response due to physical effects which are represented in the model structure can be reproduced by the simulation. This requires a detailed modelling of the different suspension elements, the wheel-rail contact and structural properties. However, a more detailed structure of the model also increases the complexity and the number of model parameters. A correct choice of these parameter values is the second requirement for a correct prediction of the real vehicle behaviour. The nominal values of the suspension parameters and admissible tolerances are defined in the manufacturer specification. When actual parameter values of a certain vehicle are needed for example for condition monitoring applications the situation is more difficult. The identification of the parameter values is therefore of large interest for the applications outlined above. This work discusses the approach of obtaining the parameter values from measurement data using identification methods. Parameter values are thus obtained for a particular vehicle and measurement condition. The variability of vehicle and wheel-rail contact parameters for several vehicles of the same type is not subject of this work. The improved calibration of the vehicle model based on the running conditions met during the measurements including variable speeds and curve radii is nevertheless the requirement for using the model in further-ranging stochastic analysis.

The following paper is structured in 4 sections. In section 2 the simulated and measured vehicle responses are compared and analysed qualitatively allowing to characterise the system and the validity range of the model. Based on these results the identification problem is designed. Its characteristics are outlined in section 3. The results of the parameter identification including the sensitivity analysis and optimization problem are presented and discussed in section 4 followed by the conclusions.

\section{Measured versus simulated vehicle response: a first qualitative comparison showing the importance of nonlinear and transient effects}

The vehicle considered is a TGV Duplex train for which acceleration and force measurements have been performed on the TGV east line. The TGV Duplex is a two-level high speed train composed of two traction units and eight passenger coaches as shown in figure 1. Like all TGV train types the coaches of the TGV Duplex are equipped with Jacobs bogies. Figure 2 shows the connection between coaches with the Jacobs bogie. This tight coupling between the coaches determines 


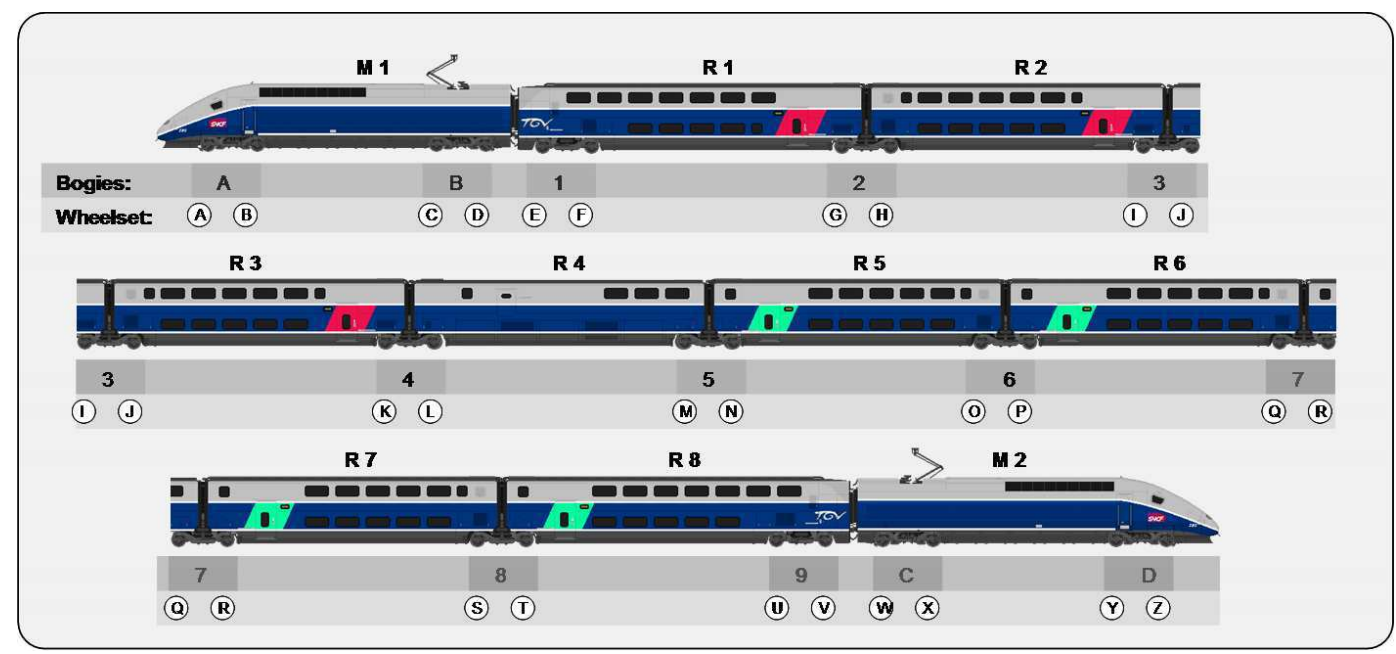

Figure 1. TGV Duplex train composed of 2 traction units and 8 passenger coaches

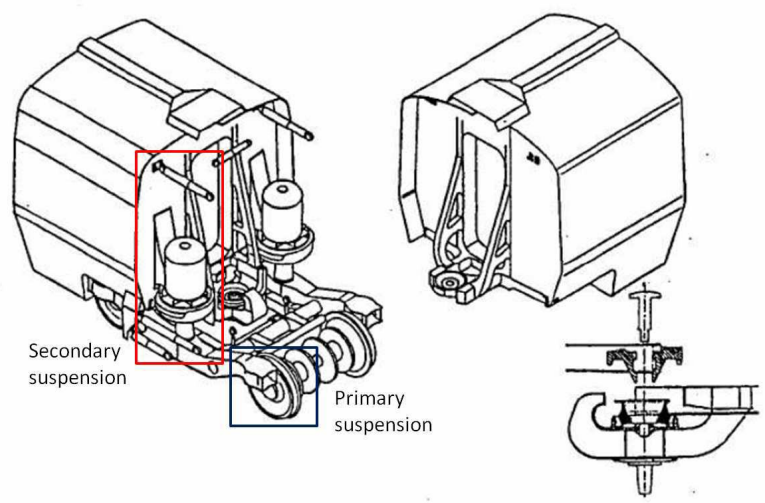

Figure 2. Jacobs bogie in a TGV train

the dynamic behaviour of the train as outlined for example in [5].

The model used is a multi-body model defined in the commercial software Vampire [6]. It consists of rigid bodies which are connected by suspension elements. The wheel-rail contact is modelled using the Hertzian law in normal direction and the nonlinear Kalker law for the computation of the lateral friction forces. The wheel-rail contact parameters are calculated and tabled as functions of the lateral wheelset displacement and the yaw rotation for defined wheel and rail profiles before the simulation. Detailed information about the rolling contact can be found in the work of Kalker [7, 8].

The track geometry is measured by the IRIS 320 measurement train [9] for track defects with wavelength between 3 and 200m. IRIS 320 is an inertial track geometry measurement system installed in a TGV train.

The vehicle track system is nonlinear due to the wheel-rail contact and nonlinear suspension elements. For the definition of the identification problem in the next section it is therefore necessary to detect and characterize these nonlinearities and their impact on the vehicle behaviour. In [10] a good summary is given about different methods and the current state of research. 

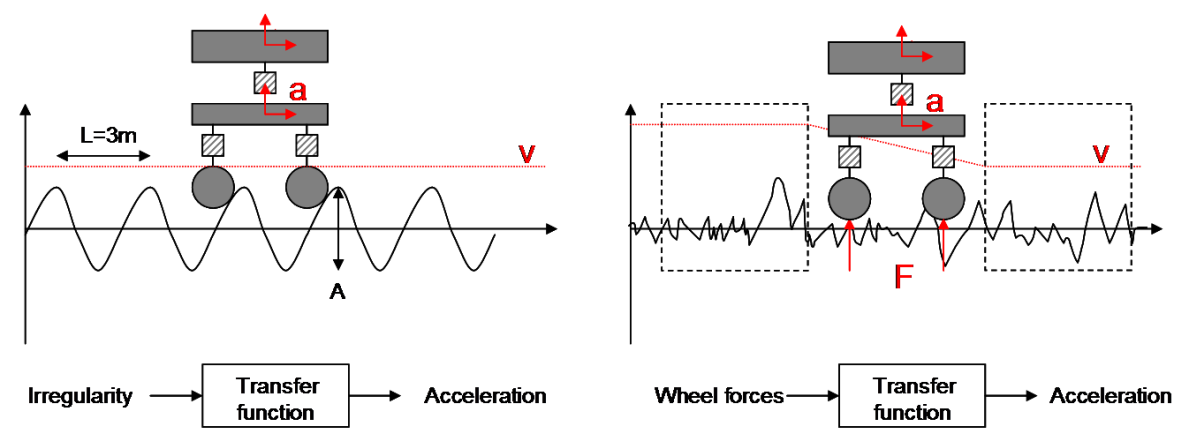

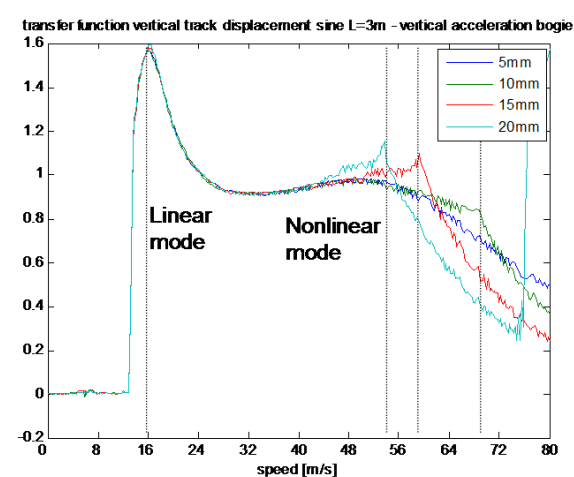

(a)
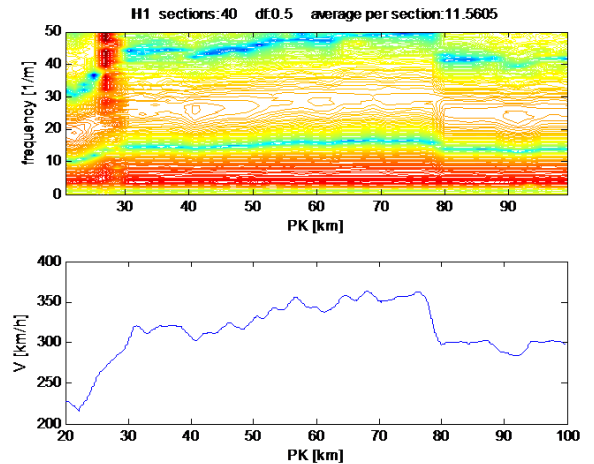

(b)

Figure 3. Calculation of the transfer function for the model with analytical track excitation of different amplitudes (a) and calculation of the transfer functions from measurement data for the TGV train under real track excitation (b)

In the sections 2.1 and 2.2 the analysis of transfer functions for different excitation conditions is used. Figure 3 illustrates the computation of the transfer functions for the measurement and the model of the TGV train.

\subsection{Transfer functions obtained by the simulation model}

For the model, the transfer functions for the fully nonlinear system have been calculated using artificial sinusoidal track defects with different amplitudes. The wavelength of the track defects corresponds to the axle-distance of the bogies such that the two wheelsets of the bogie are moving in phase. This allows to avoid any rotational movement of the bogie therefore reducing coupling effects. By increasing the velocity stepwise the transfer functions between track defect amplitude and vehicle response have been calculated for a frequency range between 0 and $20 \mathrm{~Hz}$. It can be seen that the eigenfrequencies depend on the track defect amplitude. Figure 4 shows the transfer function of the primary suspension between the track irregularities and bogie frame accelerations, in the traction unit, for different amplitudes of the track irregularities. Besides, it is not possible to superpose the response due to vertical and lateral track irregularities. The transfer functions for combined excitations show a different shape indicating a coupling between lateral and vertical behaviours. In figure $4 \mathrm{~b}$ the transfer function of the primary suspension is considerably larger for a combined lateral and vertical track excitation (case 3 in red). 


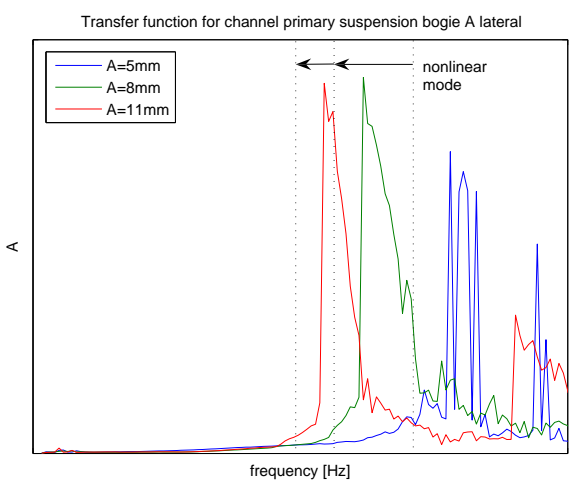

(a)

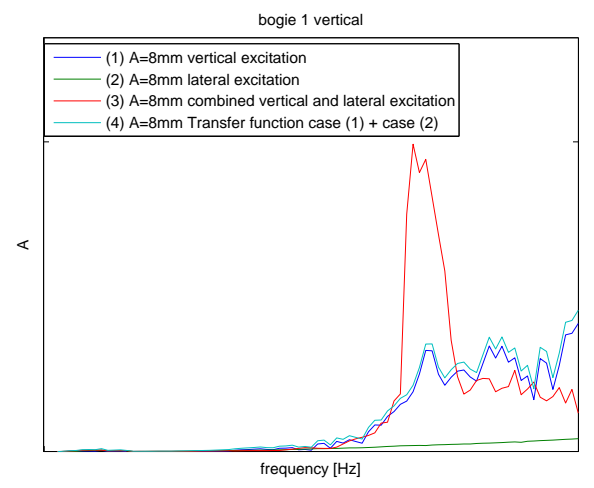

(b)

Figure 4. Transfer function of the primary suspension between the track irregularities and the bogie frame acceleration in the traction unit in lateral direction for three different track amplitudes A (a) and for vertical (1), lateral (2), combined (3) and superposed (4) sinusoidal track excitation for a track amplitude of $\mathrm{A}=8$ $\mathrm{mm}(\mathrm{b})$

\subsection{Transfer functions obtained by inline measurement data}

For the real vehicle an experimental modal analysis with defined excitation signals is not available. Therefore the inline measurements are used for an operational modal analysis. The transfer functions are calculated using autospectra $S_{x x}$ and crossspectra $S_{x y}$ between the forces (x) in the wheel-rail contact and the accelerations (y) in bogie and car body. The $\mathrm{H} 1$ estimation of the transfer function, compensating for noise on the acceleration measurement, is defined as:

$$
H_{1}=\frac{S_{y x}(f)}{S_{x x}(f)}
$$

If the spectra are calculated for the whole measurement signal nonlinear effects are averaged out. In order to analyze the dependence of the eigenfrequencies on the excitation the transfer functions are calculated for short sections along the line. The choice of the section length has to be adapted to the nonlinear effect one is interested in. Very short sections emphasize the effect due to unsteady track defects. By increasing the section length these transient excitations are averaged out and nonlinear effects due to different vehicle speeds become visible. Since the wheel forces increase with increasing speed as shown in figure 5 the analysis of the transfer functions between forces and accelerations is a suitable way to analyze nonlinearities in the vehicle suspension.

Figure $6 \mathrm{~b}$ shows the transfer function calculated from measurement data for the primary suspension. The result is compared to the transfer function obtained for the model (a). It can be seen that the eigenfrequencies depend on the vehicle speed thus on the excitation level. This leads to the conclusion that nonlinearities of the wheel-rail contact and suspension elements have an influence on the vehicle response which can not be neglected. In particular in lateral direction nonlinear effects are observed while in vertical direction a fairly linear behaviour is found.

Based on the analysis of the simulated and measured vehicle responses, the identification problem is designed in the following section. The results to be pointed out are the non-negligible nonlinear vehicle behaviour and the unsteady excitation 

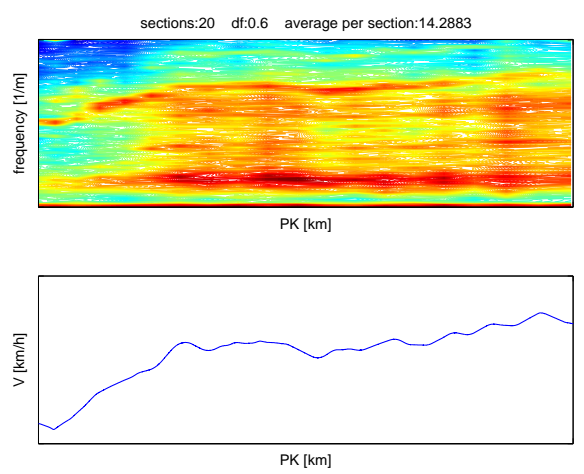

Figure 5. Short Time Fourier Transform of wheelset forces in bogie A in vertical direction for increasing vehicle speed

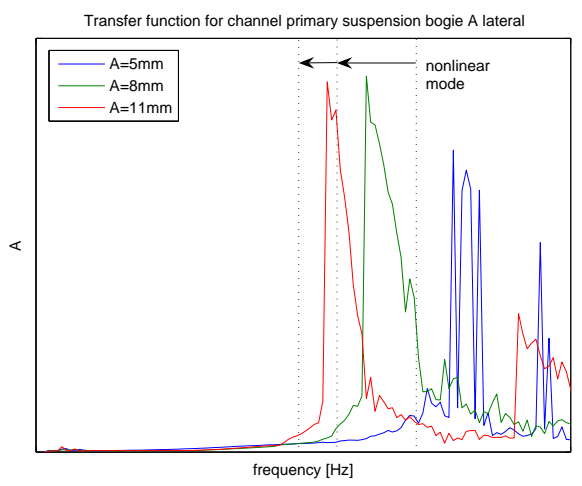

(a)

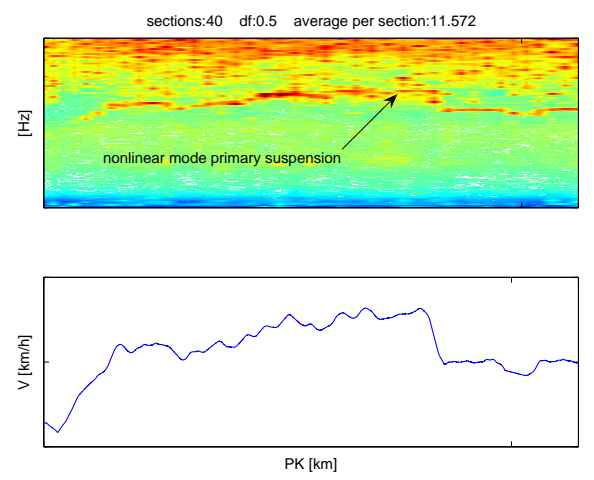

(b)

Figure 6. Transfer function of primary suspension in bogie A in lateral direction: sine excitation at three different amplitudes A for the model (a) and transfer functions calculated per section for the measurement data (b)

due to track defects.

\section{Peculiar aspects of the design of the identification problem}

The aim of the parameter identification process illustrated in figure 7 is to adjust the model parameters so that the simulation reproduces the dynamic response of the real vehicle with enough accuracy. It has been proposed in [11]. The identification process is based on inline measurement data from the TGV train running on the real track. In the TGV, accelerations have been measured in all bogies and car bodies. Besides, wheel forces are available for two measuring wheelsets.

The parameters to be identified are stiffness and damping parameters of the primary and secondary suspension as well as masses and inertia. The number of parameters describing a suspension element depends on the model, ranging from a simple equivalent stiffness to a complex physical model.

The identification of the parameters of the multi-body model is an inverse problem with particular properties. The direct problem is itself nonlinear due to the wheel-rail contact and nonlinear suspension elements. The large number of parameters leads to complicated solution surfaces and non-unique solutions. A 


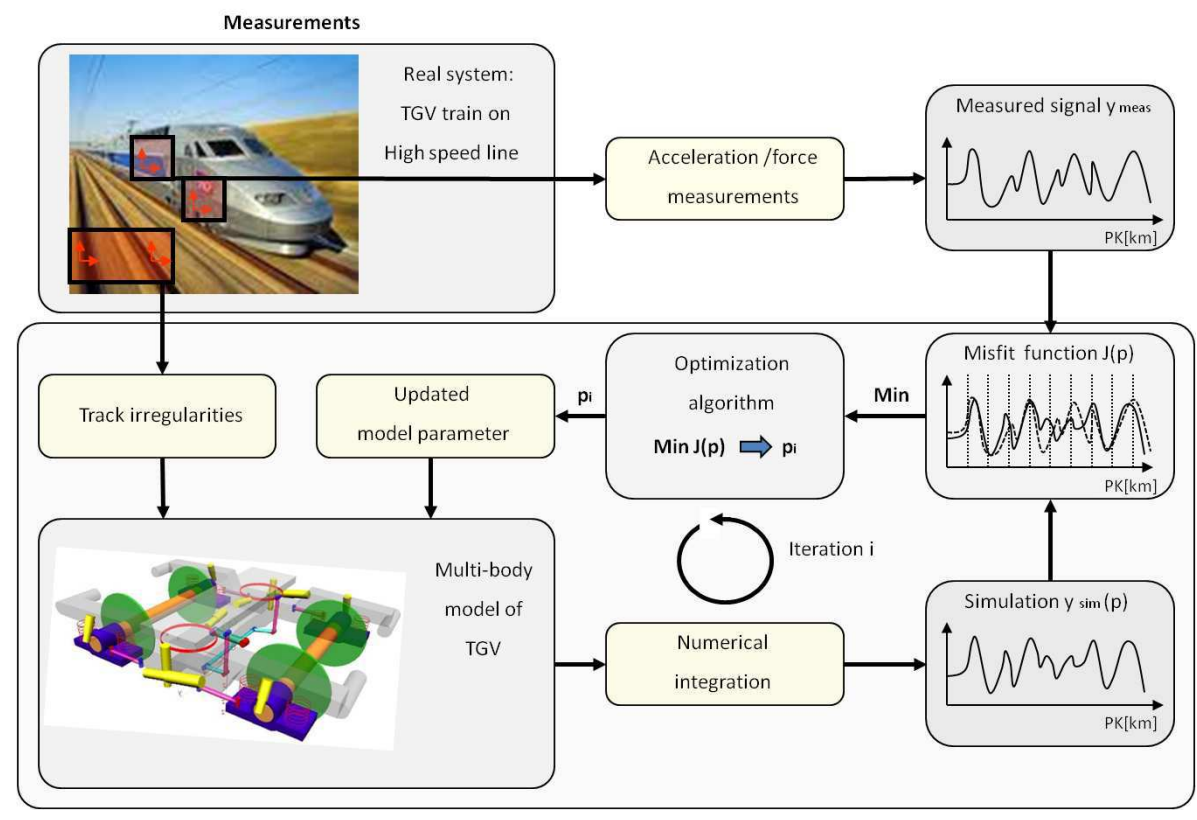

Figure 7. Schema of the parameter identification process

regularization of the inverse problem and identification methods which can deal with these conditions are required.

In the following peculiar aspects of the identification process are discussed.

\subsection{A time domain misfit function}

The misfit function describes the distance between the measurements and the result of the simulation. It can be defined in different ways and is a crucial part of the identification problem. The choice of the misfit function depends on the characteristics of the available experimental and simulation data. It can be defined either in the time domain or in the frequency domain. For linear systems the definition in the frequency domain has many advantages. It uses the modal parameters (eigenfrequencies and eigenmodes) thus allowing to describe and compare the system behaviour by a relatively small number of parameters.

For nonlinear systems these modal parameters are not directly available. They are not system-inherent but depend on the excitation. The principle of superposition does not apply. The speed dependent eigenfrequencies of the transfer functions and the result when superposing vertical and lateral excitation, analysed in section 2, illustrate this effect. Nevertheless approaches using the principle of modal analysis for nonlinear systems have been developed. The principle of normal modes for undamped linear systems has been transferred to nonlinear systems in [12-14] by defining nonlinear normal modes (NNM). Characteristic properties of these modes are the observed frequency-energy dependence and the modal interaction. The eigenfrequencies depend on the energy brought into the system, being here related to the vehicle speed and the track geometry quality. Internal resonances in the system lead to an energy transfer from the excited mode to other nonlinear modes explaining the presence of several eigenfrequencies in the response spectra. 
From the transfer functions presented in the previous section it is known that nonlinearities have an important effect on the dynamic behaviour of the vehicle, notably in lateral direction. Due to varying vehicle speed and unsteady excitation by track irregularities, a linearisation for a certain working point and nonlinear normal modes are not applicable. Therefore the misfit function is defined in the time domain using acceleration and force signals.

Differences between measurement and simulation can be either due to an irrelevant choice of the model, neglecting important physical effects of the real system, or due to wrongly chosen parameter values. In the latter case, by minimizing the difference between model and measurement the parameter values can be adjusted. In order to minimize the misfit function a global optimization method is used which will be discussed in section 4 .

The time-domain misfit function can be defined in different ways. Most commonly the least-square distance between the signals is calculated and integrated over the signal length. If the measurement signal is corrupted by noise methods which take into account the noise level can be used. The maximum-likelihood method [15] takes into account prior information about the measurement noise in the misfit function definition. The correlation-based method [16] uses the effect that noise signals are not correlated after a short time while the vehicle response signals are still correlated. This can be shown by calculating the auto-spectra of the signals. For vehicle dynamic purposes only low frequencies are taken into account. Elastic body modes are neglected allowing the use of rigid bodies. Therefore the acceleration signals are filtered at low frequencies up to $20 \mathrm{~Hz}$. In this frequency range the noise level is low and can be neglected. The least-square method is therefore sufficient and used in the following. For every time step the error vector $e(p, t)$ between model response $x_{\text {model }}(t, p)$ and measured response $x_{\text {meas }}(t)$ is computed by $e(p, t)=$ $x_{\text {meas }}(t)-x_{\text {model }}(t, p)$. Using the square of the $L_{2}$ norm and a weighting matrix $\mathrm{W}$ the misfit function value is calculated and integrated over the time:

$$
J(p)=\frac{\int_{0}^{T} e^{T}(p, t) W e(p, t) d t}{\int_{0}^{T} x_{\text {meas }}^{2}(t) d t}
$$

Since one is interested in the distance relative to the measured vehicle response, the misfit function is normalized with the integral over the squared measurement signal.

The frequency range of the vehicle response for which the misfit function is defined depends on the track excitation spectrum and the eigenmodes of the vehicle which are correctly represented by the model. For vehicle dynamics purpose the spatial track excitation spectrum is transformed into a time spectrum. It depends therefore both on the measurement range of the track geometry measurement system as well as the speed of the vehicle. On high speed lines in France the track geometry is measured for wavelengths between 3 and $200 \mathrm{~m}$. For a vehicle speed of $300 \mathrm{~km} / \mathrm{h}$ this corresponds to an excitation frequency range between 0.4 and $30 \mathrm{~Hz}$. The valid frequency range of the model is defined by comparing measured and simulated transfer functions. For the example of the vertical bogie acceleration it can be seen in figure 8 that the model reproduces well the dynamic behaviour for the real system for a frequency range between 1 and $14 \mathrm{~Hz}$ while for higher 


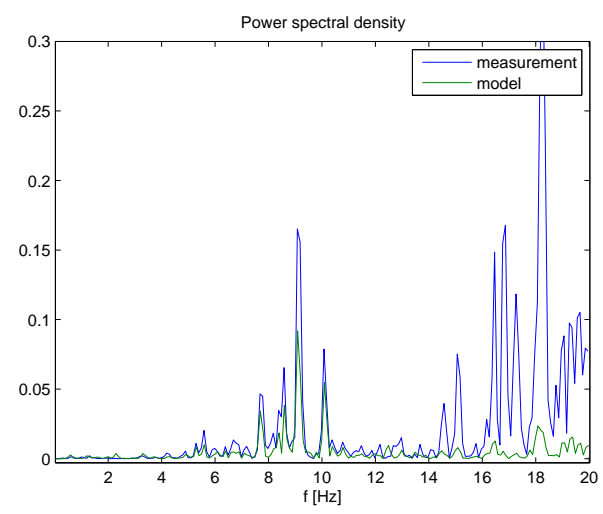

Figure 8. Measured and simulated power spectral density of bogie A acceleration in lateral direction

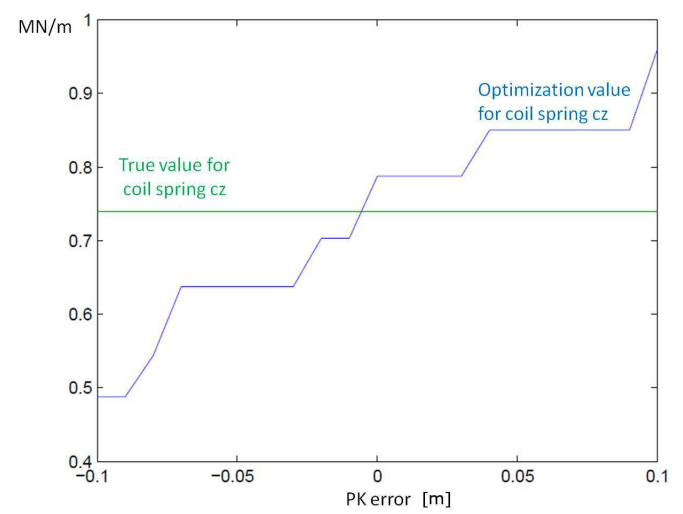

Figure 9. Influence of the error of the kilometric position $(\mathrm{PK})$ on the identification result for the coil spring stiffness $\mathrm{CZ}$

frequencies the model response is insufficient to account for the experimental response peaks. This is due to the fact that the elastic modes at higher frequencies are not reproduced by the model built up from rigid bodies.

\subsection{Synchronisation of measurement and simulation}

The definition of the misfit function in the time domain requires that the measured and the simulated signals are synchronized. Already a small shift of the kilometric position (PK) of the signals can increase the misfit function significantly and lead to identification errors. In order to analyze the effect of a kilometric shift between measurement and simulation, parameters of the TGV model have been identified using a reference simulation as "virtual measurement data". In the case of zero shift the exact value of the true parameters is identified. Then the identification has been repeated for increasing shifts between the signals. In figure 9 it can be seen that small shifts lead to important errors in the identified parameter values. An efficient synchronisation is therefore crucial for the identification problem.

In order to synchronise measurement and simulation signals the cross-correlation between the measured vertical axle-box accelerations and the vertical track irregularities is used. Since the axle-box acceleration is measured below the primary suspension thus avoiding the effect of nonlinearities a very good correlation is obtained. The twice integrated axle-box acceleration is used nearly as a sensor for the 


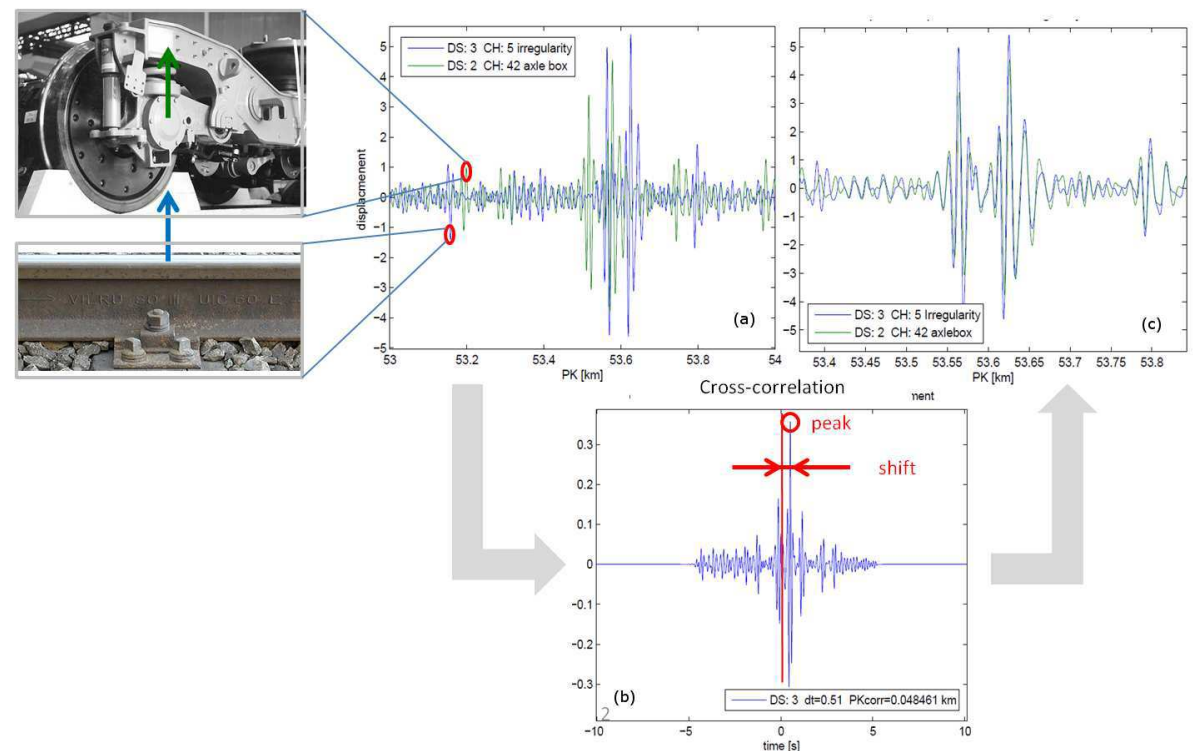

Figure 10. Correction of the kilometric position (PK): time signal of the track irregularity and axlebox acceleration signal before correction (a), cross correlation function between vertical track irregularity and vertical axlebox acceleration (b), corrected time signal (c)

vertical track defect. If the correlation is sufficiently good the synchronisation can also be performed directly between measured and simulated acceleration signals. The PK correction is illustrated in figure 10.

\subsection{Evaluation of the misfit function with initial parameters taking into account the correlation with track geometry and design}

The misfit function can be composed by one or several signals over a defined length. For the TGV train wheel forces as well as accelerations from bogies and car bodies are available. The correlation between measurement and simulation depends on the considered signal. For each signal the calibration of the initial model aims at analysing the behaviour of the TGV model for the initially chosen parameter set. The frequency and the time response of the model are compared to the measured data. This is done by calculating the misfit function for sections of a defined length $(125 \mathrm{~m})$. Due to variations in the kilometric shift along the line, the measurement and simulation signals are synchronised for each section separately. If the coherence is sufficiently good, the cross-correlation is applied in order to obtain the shift between the signals. In the case where the coherence does not allow to apply the synchronisation, the kilometric shift is interpolated from the surrounding sections.

Figure 11 shows the misfit function for the vertical force and the lateral acceleration in bogie $\mathrm{A}$. In both cases the misfit function indicates a satisfactory correlation between measurement and simulation. However, it is observed that the misfit function varies considerably between different sections. For the vertical wheel force, values between $10 \%$ and $70 \%$ and for the lateral acceleration values between $40 \%$ and $140 \%$ are obtained. How can this significant difference be explained? One approach is to analyse the correlation between the track geometry and design on one part and the misfit function on the other part. This might allow to answer the question if the capacity of the model to reproduce the real vehicle behaviour depends on the track quality and other running conditions. 


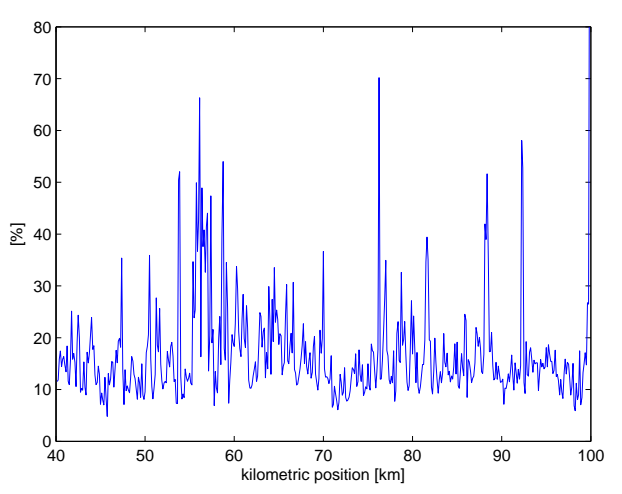

(a)

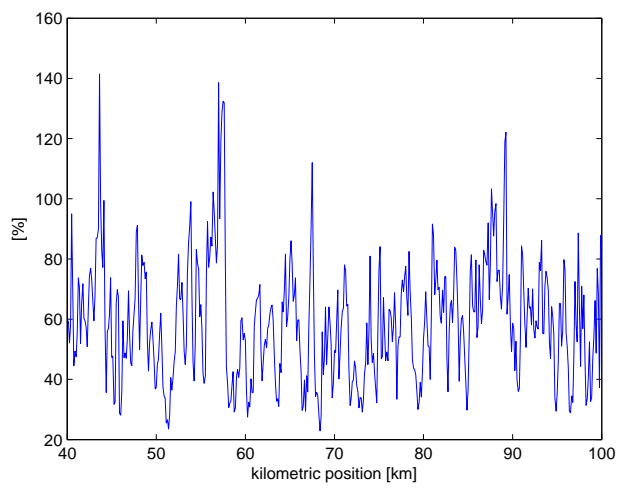

(b)

Figure 11. Initial misfit function for the vertical wheel force (a) and the lateral acceleration (b) in bogie A for sections of $125 \mathrm{~m}$ length

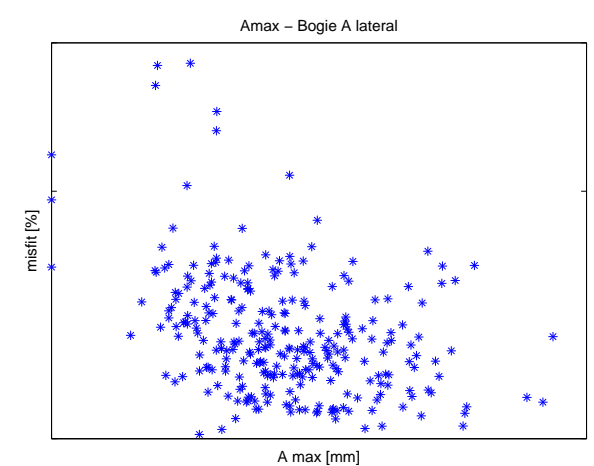

Figure 12. Correlation between the maximal cross-level defect amplitude Amax with the misfit function per section in bogie A lateral

Figure 12 shows the correlation between the maximal amplitude of the cross-level track defect per section and the misfit function for the lateral acceleration in bogie A. Even though no important correlation is observed it is found that the misfit function is lower for higher track defect amplitudes. One possible explanation is that for sections with very low irregularity levels the vehicle response is controlled by other excitations, aerodynamic forces for example, or the eigendynamics of the vehicle.

In figure 13 the correlation with respect to the track curvature and cross-level offset is shown. It can be seen that low misfit function values appear in straight track (curvature $=0$ ) while the highest values appear in curves. The model reproduces better the behaviour on a straight track.

In general, the misfit function is worse for the lateral direction. While the misfit function for the vertical wheel forces is mainly around $15 \%$ the lateral forces are worse reproduced by the model with misfit function values between $40 \%$ and $90 \%$. Finally it is observed that the misfit function has higher values for accelerations measured above the secondary suspension.

The results obtained from the evaluation of the initial misfit function comply with 


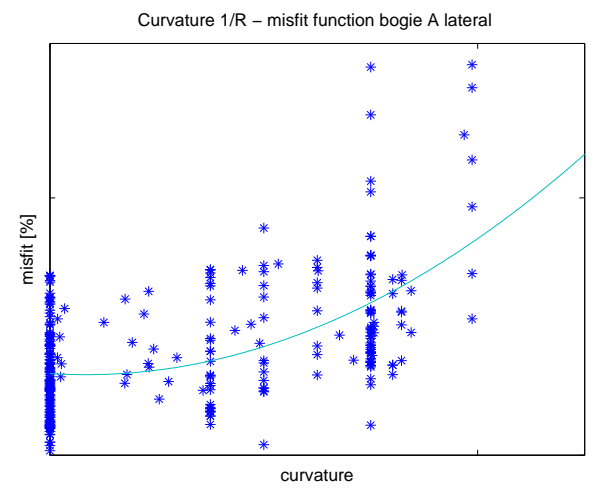

(a)

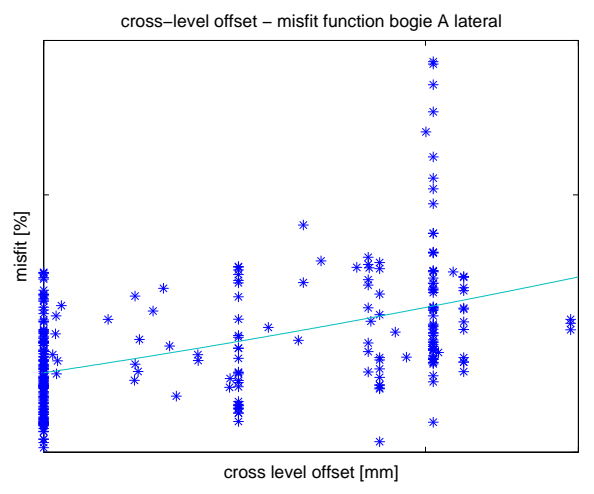

(b)

Figure 13. Correlation of the misfit function for the lateral acceleration in bogie A with respect to the curvature (a) and cross-level offsets (b)

expectation: due to the nonlinear characteristic of the wheel-rail contact in lateral direction, the correlation is worse for lateral than for vertical vehicle responses. The same applies for responses in the car body compared to the bogie or axleboxes. The secondary suspension with its nonlinear airspring in coaches reduces the correlation between model and measurement.

\section{Identification of a reduced set of the most relevant parameters obtained from an initial sensitivity analysis}

The TGV model is described by a large number of parameters. If all suspension element parameters are considered independently more than 1000 parameters have to be taken into account in the identification problem. If parameters of the wheel-rail contact are also considered the number increases even more. It is therefore crucial to select the most relevant parameters for the identification problem. The choice of the parameters depends on several factors: the knowledge about the parameter value in the real system, the influence of the parameter on the vehicle behaviour, the aim of the analysis and the available vehicle responses. In general, one can only identify parameters which have an influence on the vehicle responses which are considered in the misfit function. The identifiability of the model parameters depends therefore on the degrees of freedom measured in the real system and their coupling.

A priori knowledge about the suspension parameters can be obtained from test rig measurements. In some cases the suppliers give information about the suspension element characteristics and tolerances. If a parameter is exactly known it is not included in the identification problem. The influence of parameters which can vary within tolerances is studied using a sensitivity analysis. Since the influence of one parameter depends in general on the other parameter values, global methods taking into account the interaction between the parameters values have to be used.

\subsection{Initial sensitivity analysis with Morris method}

The Morris method [17] is a one-factor-at-a-time method which can take into account nonlinearities and interactions between the parameters. The method 


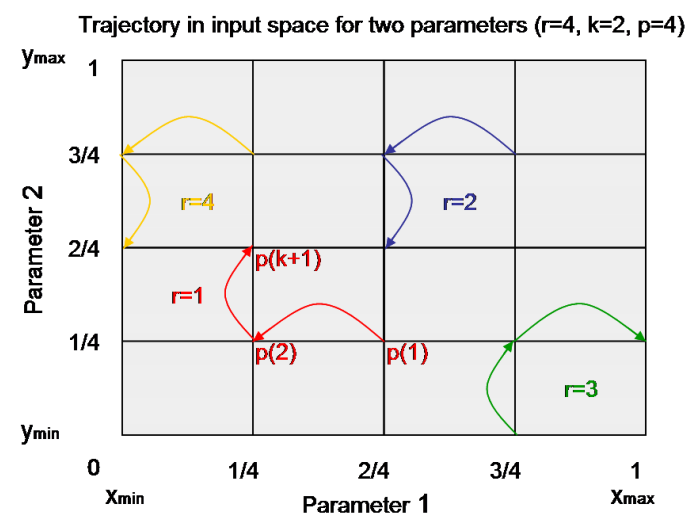

Figure 14. Sampling for Morris method with two parameters $\mathrm{x}$ and y discretized by 5 levels and 4 samples $(\mathrm{k}=2, \mathrm{p}=5, \mathrm{r}=4)$

proposes two estimates: one which represents the overall effect of the parameter and another estimating the second-and higher-order effects of the parameter.

The basic idea of the Morris method is to repeat a local one-factor-at-a-time method several times for randomly chosen data sets. This allows to take into account the interactions between the parameters. The one-factor-at-a-time method describes the influence of one parameter on the misfit function $J$ when the others are fixed.

The calculation of this so-called elementary effect is repeated for samples of the parameter set $\mathbf{p}$ giving a distribution of elementary effects for each parameter. The samples are chosen randomly according to the frequency distribution defined for each parameter. The average of the distribution of elementary effects describes the overall effect of the parameter and its standard deviation is a measure of the nonlinearity and the interaction between the parameters. A high value of the standard deviation means that the elementary effect of the parameter depends strongly on the values of the other parameters. If the interaction is low the elementary effect is not influenced by the other parameters and the standard deviation is small.

The cost needed for the computation of the sensitivity is an important criterion. Basically, if $r$ samples are calculated for a set of $n$ parameters the number of misfit function evaluations is $2 n r$. Morris proposes an approach which reduces the computational cost compared to Monte-Carlo calculations.

The samples of the parameter vector are constructed outgoing from an initial vector. The following samples are obtained by changing only one component with respect to the previous vector. The component is randomly selected among all parameters apart the ones which have been varied before. Therefore two consecutive samples differ only in one component and each component has been varied at least once. The parameters are varied using a small number of values which are chosen within the range of tolerance. Figure 14 illustrates the sampling procedure for the case of two parameters $(\mathrm{k}=2)$ and 5 value levels $(\mathrm{p}=5)$. The sampling procedure is repeated 4 times $(\mathrm{r}=4)$. For more details it is referred to [17].

For each misfit function the sensitivity of the parameters is obtained in form of a point distribution with the overall effect indicated on the $\mathrm{x}$ - and the coupling and nonlinear effect on the y-axis. A first example is shown in figure 15 for the 


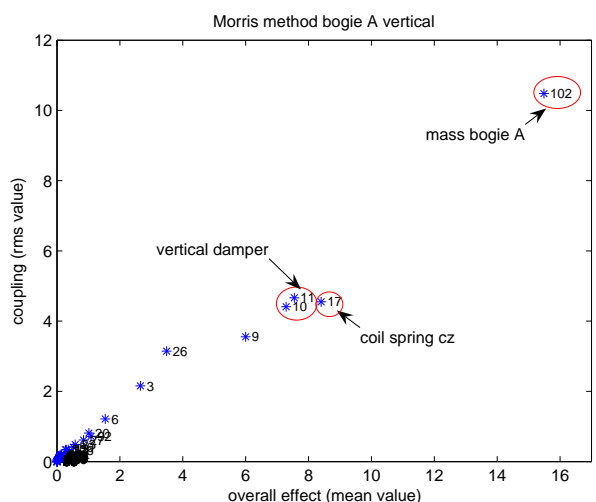

(a)

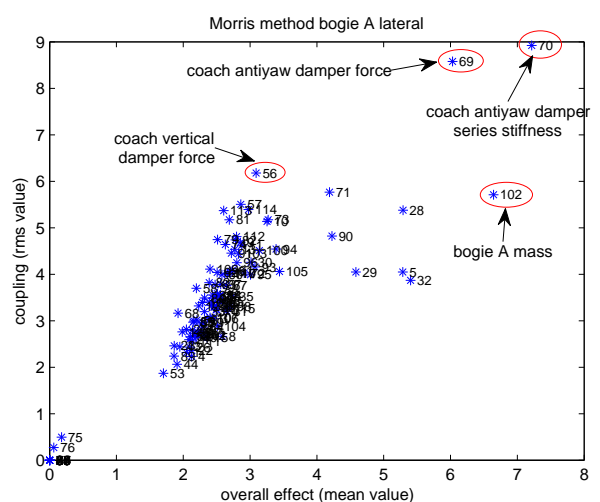

(b)

Figure 15. Morris method for bogie A in vertical (a) and lateral direction (b)

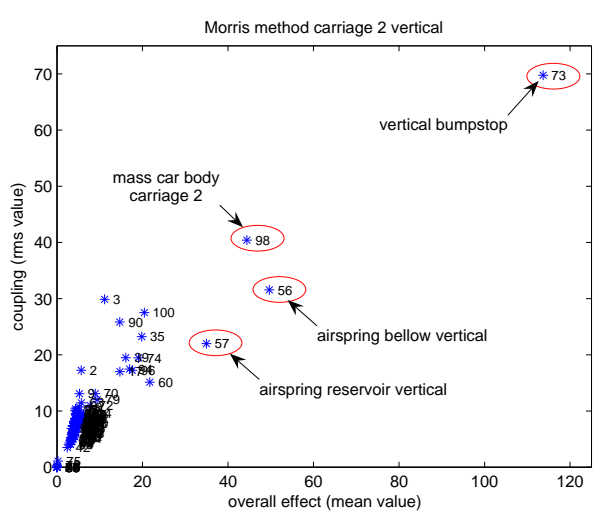

(a)

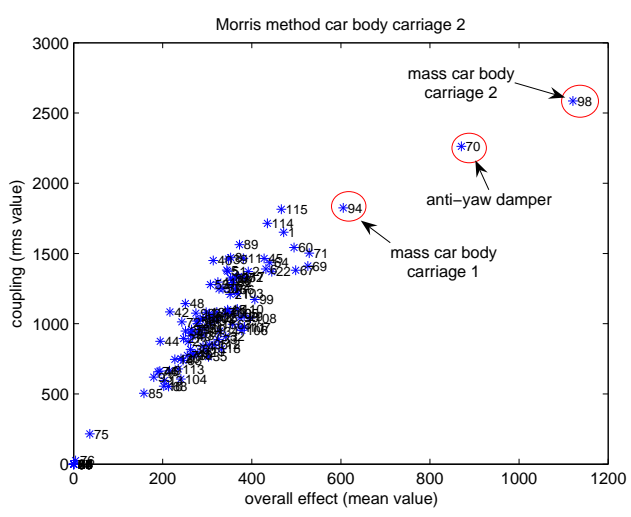

(b)

Figure 16. Morris method for carriage 2 in vertical (a) and lateral direction (b)

bogie $\mathrm{A}$ in the traction unit in lateral and vertical directions. It is found that in vertical direction only vertical suspension parameters of the traction unit have an influence (vertical damper, coil spring, etc.) The interaction and nonlinear effects between them are important. All other parameters have a negligible influence indicating that the coupling from the carriages to the traction unit and the lateral parameters to the vertical vehicle response is low. In lateral direction on the contrary important nonlinear and coupling effects between a large number of parameters are found with the anti-yaw damper as an element which is particularly important. In both cases the bogie mass has an outstanding influence.

As a second example the car body acceleration of carriage 2 is considered in figure 16. In general, the influence of the parameters on the lateral response is much higher. The interaction and/or nonlinear effects of a large number of parameters are important. The vertical response is less sensitive to parameter changes and controlled by few parameters notably the airspring.

For a better understanding of the sensitivities and interactions the results of the Morris-method are visualized in two parameter-misfit function tables: one for the overall effect and another for the coupling. Each value in the table represents the overall effect or coupling of one vehicle parameter relative to one misfit function. 


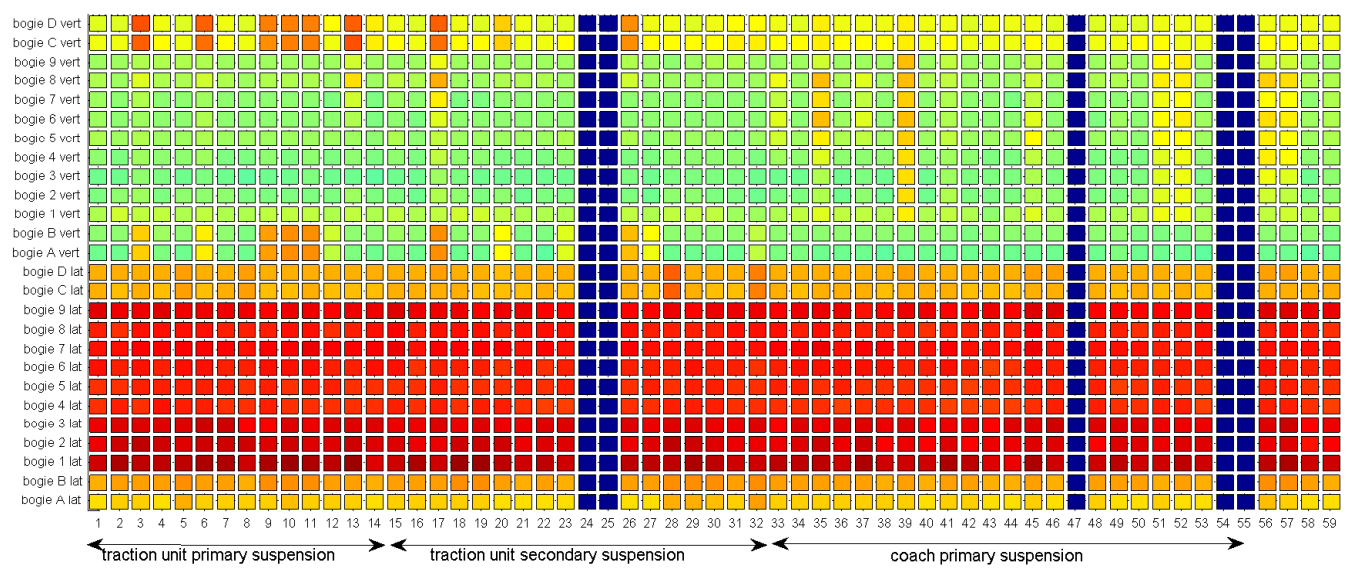

(a)

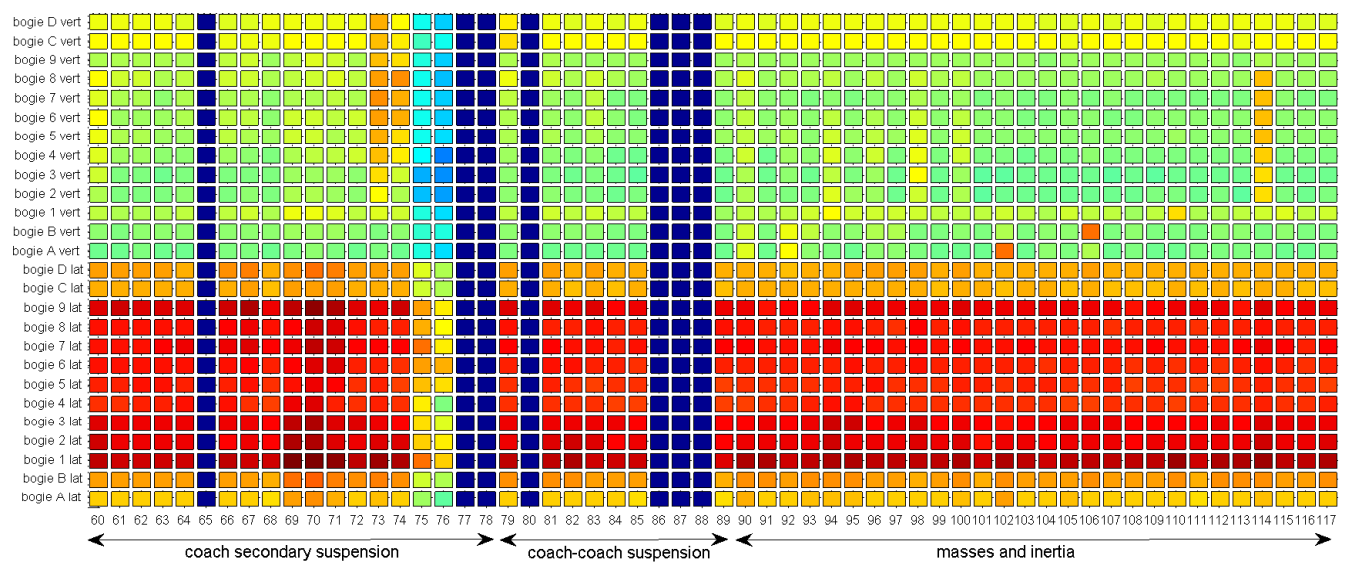

(b)

Figure 17. Morris method for the bogies in vertical and lateral direction for the overall effect using real track excitation for 117 vehicle parameters (a:1-59, b:60-117)

Figure 17 shows the overall effect of 117 parameters on the bogie misfit functions. Information about the real correlation of the parameter values is not available. In order to limit the number of parameters it is supposed that all elements of the same type have the same parameter value. The correlation factor between the parameters of the same element type is considered to be one.

Based on the results of the sensitivity analysis, the vehicle parameters acting as input parameters to the identification problem are chosen. Either these parameters are selected for a previously defined misfit function or the misfit function is designed allowing to identify certain vehicle parameters.

\subsection{Necessity of global optimization}

Parameter identification aims at adjusting the parameter values by minimizing the misfit function. This requires the implementation of an optimization algorithm. The choice of a suitable optimization algorithm depends on the characteristics of the misfit function solution surface. If the solution function is convex and has only one minimum in the defined parameter range local methods can be used. These methods are deterministic and show a fast convergence. Most commonly they use 
the first and second derivatives of the misfit function. Local methods are outlined in the books of Nocedal [18] and Geiger [19].

For optimization problems where the misfit function has a non-convex solution surface with several local minima the use of local optimization methods can be inappropriate. They converge to the local minimum of the attractor region where the initial parameter set is situated. In this case global methods have to be used. They avoid the convergence to the closest local minimum by the use of probabilistic operators allowing to leave the attractor region of a local minimum. One drawback of these methods is the high number of iteration steps required.

Global methods are often based on probabilistic operators. In the simulated annealing method [20] the probability for the acceptance of an increase of the misfit function value depends on a parameter called temperature. The method simulates the cooling process of a material. Another widely-used approach comprises the genetic algorithms discussed in [21]. They are based on the principles of evolution and provide a set of possible solutions.

Due to the complexity of the misfit function solution surface of the TGV model the global simulated annealing method and a genetic algorithm have been applied in this work.

Track and vehicle measurement data are available for the whole TGV east line of around $300 \mathrm{~km}$ length. An optimization using all these data or a large part of it is impossible due to the computational cost of the simulation and the high number of iterations of global optimization algorithms. It is therefore necessary to define learn sections for which the optimization is performed. A learn section represents a short section of the line for which the simulation is performed and the misfit function calculated at each iteration of the optimization. In this work learn sections of $1 \mathrm{~km}$ length are used. A valid identification of the parameters requires that the results obtained from the learn sections are representative for the whole line and that several learn sections in different running conditions give the same result. Differences would indicate the identification for a certain working point and an insufficient model structure.

From the calibration of the initial model it was found that the track design has an important influence on the misfit function. It is therefore reasonable to define one learn section on a straight track and another learn section on a curved track. The speed for both sections is $300 \mathrm{~km} / \mathrm{h}$.

\subsection{Application examples}

For the two defined learn sections different optimization configurations have been tested. They are summarized in table 1 . The parameter identification has been applied to three misfit functions with increasing complexity using the global simulated method and a genetic algorithm as well as the local Pattern Search method. The first misfit function is defined by only one signal, the vertical acceleration in bogie A. In the second application case all accelerations of one vehicle are used, thus including the vertical and lateral accelerations in the bogie and car-body. Finally, the third misfit function takes into account accelerations measured in the traction unit as well as the first and second coach. The tolerance 
Table 1. Reduction of the misfit function and final value for local and global optimization methods and three identification problems with increasing complexity

\begin{tabular}{lllll}
\hline & & \multicolumn{3}{c}{ Reduction misfit function [\%] (final value) } \\
\hline \multicolumn{2}{c}{ Test cases } & Bogie(A) vertical & Traction unit(1) & $\begin{array}{l}\text { Traction unit(1), } \\
\text { coaches(1)(2) }\end{array}$ \\
\hline Local method & Pattern Search & $44.2(20.8)$ & $1.6(41.5)$ & $7.7(43.4)$ \\
Global method & Simulated Annealing & $49.5(18.9)$ & $11.2(39.3)$ & $14.3(40.3)$ \\
Global method & Genetic algorithm & $42.6(21.5)$ & $11.2(39.3)$ & $13.9(40.5)$ \\
\hline
\end{tabular}

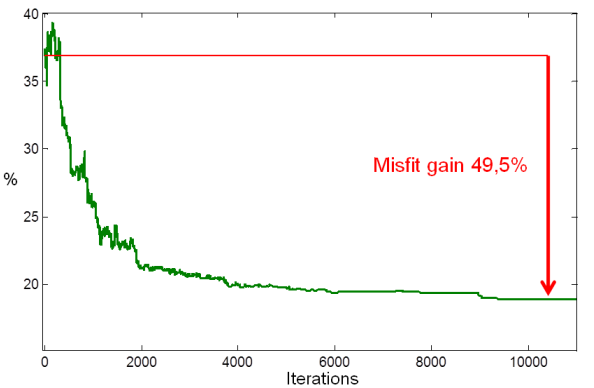

(a)

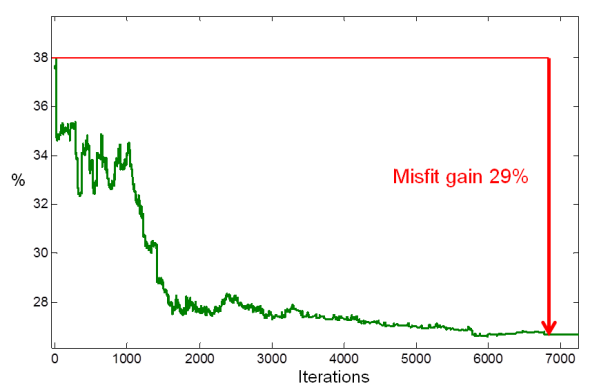

(b)

Figure 18. Reduction of the misfit function for straight (a) and curved (b) track as a function of iteration steps

of the vehicle parameters, defined by the admissible variation around the nominal parameter value, is $50 \%$.

The comparison of the local Pattern Search method with the simulated annealing method and the genetic algorithm confirms the need for global optimization. Only for the case "Bogie A vertical" the local method leads to the same result indicating a convex misfit function. If the misfit function is composed by several vehicle responses the solution surface becomes non-convex and global methods have to be used. Both the simulated annealing method and the genetic algorithm lead to the same results.

In the following two application examples with a single signal in the misfit function are discussed in detail.

\subsubsection{Example of vertical acceleration in bogie $A$}

As a first example the identification result is discussed for the vertical acceleration in bogie A and 76 vehicle parameters considered. The good correlation of the initial model and the absence of nonlinear effects make this a simple case. Figure 18 shows the reduction of the misfit function for the learn section in straight track and in curve. In straight track the misfit function is reduced by $49.5 \%$ giving a final misfit function value of $18.9 \%$. As it can be seen from the time signal in figure 19 this corresponds to a very good correlation between measurement and simulation.

For the learn section in curved track the misfit function is only reduced by $29 \%$ giving a final value of $26.8 \%$. This confirms the result obtained from the initial calibration. Even after the optimization the model is less able to represent the dynamic behaviour of the real vehicle in curves than on straight track.

When looking at the identified parameter values, differences between straight and 


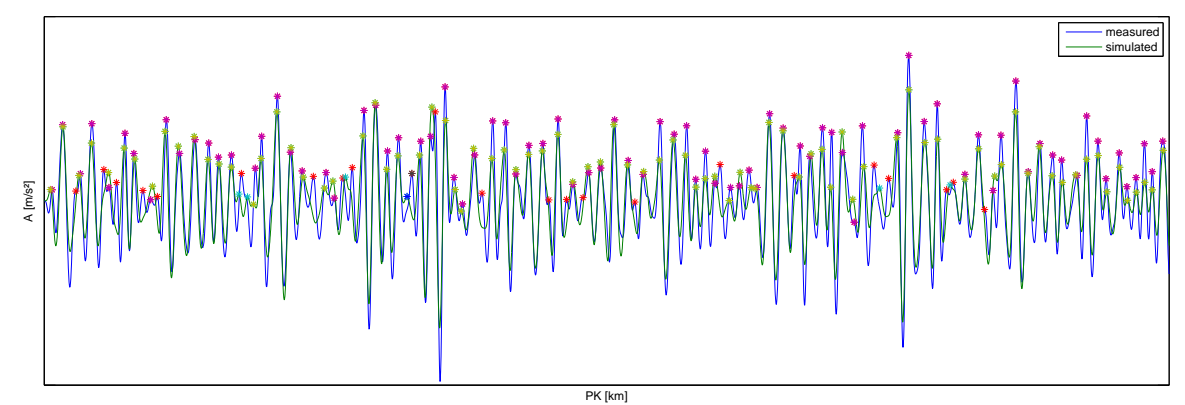

Figure 19. Measured and simulated acceleration signals for the vertical acceleration in bogie A

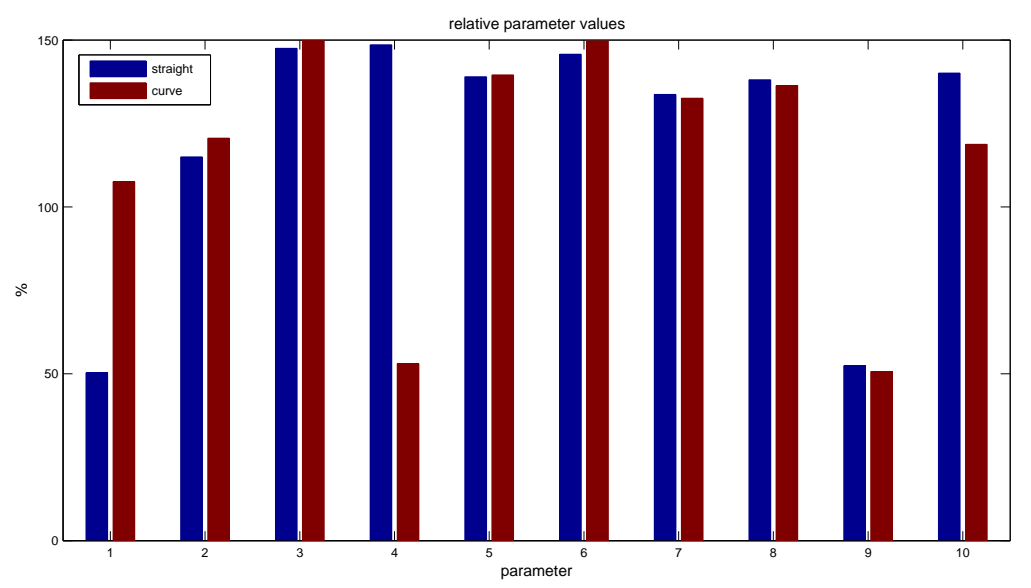

Figure 20. Relative parameter values of the primary suspension in the traction unit for straight and curved track

curved track are found also. Figure 20 shows for the parameters 1 to 10 the relative modifications. For the longitudinal stiffness of the coil (1) and the guidance spring (4) the identified values differ extremely while for the other parameters a good correlation between straight and curved track is found. This can be explained when the result of the sensitivity analysis is considered. The two mentioned parameters have a low influence on the vertical vehicle response. Their identification using only the vertical bogie acceleration is therefore not possible. For a identification of these parameters the lateral response has to be considered in the misfit function.

\subsubsection{Example of lateral acceleration in carriage 2}

In the second example the lateral acceleration in carriage 2 is used in the misfit function. Due to the secondary suspension and nonlinear effects the initial misfit function has a value of $88 \%$. From the sensitivity analysis it is found that both vertical and lateral suspension parameters control the lateral vehicle response. Therefore a large number of vehicle parameters is considered in the identification problem.

For a parameter tolerance of $10 \%$ the misfit function is reduced by $34 \%$ giving a final value of $58 \%$. An increase of the parameter tolerance to $50 \%$ increases the misfit function gain only slightly indicating the important influence of parameters on the lateral vehicle dynamics. The model is less robust and even small errors in 


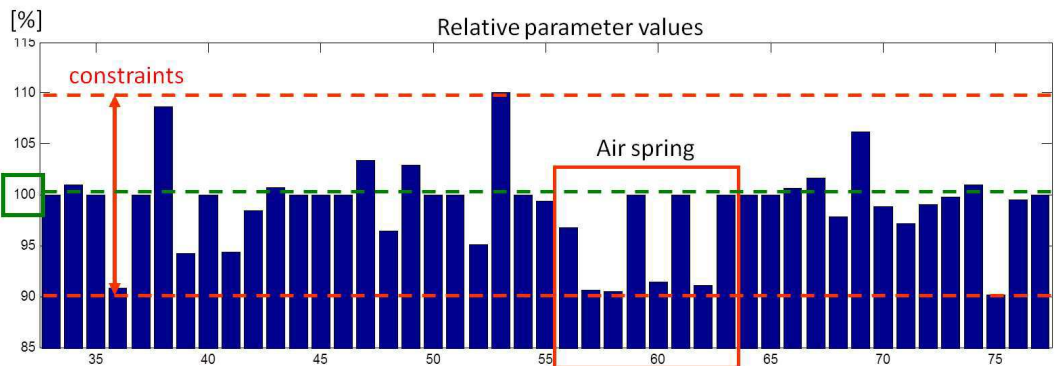

Figure 21. Relative changes of parameter values for a misfit function using the lateral acceleration in carriage 2

the parameters have a strong effect on the model performance.

In order to improve the model performance a simple linear stiffness model of the airspring has been replaced by a thermodynamic model. Figure 21 shows the relative change of the parameter values for a tolerance of $10 \%$ for this model. It can be seen that 4 of 8 parameters of the airspring reach the defined boundaries. It illustrates the difficulty when the model structure is improved by a more detailed modelling. The nominal parameters of the thermodynamic airspring model which replaced the simple equivalent stiffness are not well known and increase the complexity of the identification problem.

\section{Conclusions}

The results of the previous section show the potential of parameter identification methods for the complex nonlinear system of a railway vehicle. Measured forces and accelerations are used in order to adjust the simulation model to the real system. A good correlation between model and measurements is needed for virtual homologation and track maintenance procedures based on the vehicle response. An identification of suspension parameters could be also used for condition monitoring purposes.

The misfit function which describes the distance between simulation and measurement is defined in the time domain using least-squares. This choice was made after analyzing the excitation signals and transfer functions of the track-vehicle system. By comparing the transfer functions of the primary and secondary suspension it is observed that nonlinearities have an important influence on the lateral dynamics of the TGV train.

In the identification problem only vehicle parameters which have an influence on the defined misfit function are identifiable. Therefore a global sensitivity analysis taking into account the interaction between the parameters has been applied to more than 100 vehicle parameters. For the transfer function it revealed a difference between the vertical and lateral system behaviour. While the vertical vehicle response is controlled by a relatively small number of vertical suspension parameters located at the same position in the vehicle, an important coupling is found for the lateral vehicle response. It is controlled by a large number of parameters. This results in a more complex identification problem if the lateral vehicle response is taken into account in the misfit function. 
Taking into account the results of the sensitivity analysis several identification problems are defined. In the first case only the vertical vehicle response in bogie A is used in the misfit function. Already the initial model shows a very good correlation $(37 \%)$ and depending on the defined parameter tolerances a reduction of the misfit function between $10 \%$ and $40 \%$ is obtained. The vertical direction of the suspension is well modelled in the structure of the TGV model. In the second case the lateral response in the car body is used adding several difficulties to the identification problem: the nonlinear vehicle behaviour in lateral direction, the important coupling between vehicle parameters and the complex secondary suspension. Consequently the initial misfit function is worse (90\%). The identification process leads to an important reduction of the misfit function already for small parameter tolerances. In lateral direction the model is less robust and more sensitive to parameter errors. The persisting differences between model and measurement indicate that a more detailed model structure might be necessary. However, as shown for the example of the thermodynamic airspring model this increases the complexity of the identification problem and adds difficulties with choosing nominal parameter values.

As a perspective for future work the consideration of parameters of the wheel-rail contact is envisaged. They have an important influence on the vehicle dynamics but since exact information about the conicity and friction coefficients is often not available they add uncertainty to the simulation results. An identification of these parameters from measurement data could help to better understand the vehicle behaviour under different running conditions and improve the simulation results. If the results show an important variability for the wheel-rail contact parameter the introduction of a probabilistic model can be reasonable. The parameters are then considered as stochastic parameters with a certain probability distribution.

\section{References}

[1] CEN, EN 14363:2005, Testing and Simulation for the acceptance of running characteristics of railway vehicles - Running behaviour and stationary tests, CEN/TC 256, 2005.

[2] Yong Guo, Algorithmen zur On-board-diagnose von Fahrwerksschden an Schienenfahrzeugen, Dissertation, Technische Universitat Berlin, 2005, in German.

[3] S. Bruni, R. Goodall, T. X. Mei, H.Tsunashima, Control and monitoring for railway vehicle dynamics, Vehicle System Dynamics, 45 (7-8), 743-779, 2007.

[4] P. Li, R. Goodall, P. Weston, C.S. Ling, C. Goodman, C. Roberts, Estimation of railway vehicle suspension parameters for condition monitoring, Control Engineering Practice 15, pp. 43-55, 2007.

[5] O. Krettek, J. Grajnert, Zur luftfeder- und schwingungstechnischen Auslegung des TGV Atlantique, ZEV, Glasers Annalen, vol. 120, no8, pp. 308-325, 1996, in German.

[6] AEA Technology, Vampire User Manual, Version 4.31.

[7] J.J. Kalker, Wheel-rail rolling contact theory, Wear, 144, 243-261, 1991.

[8] - , Three-Dimensional Elastic Bodies in Rolling Contact, Kluwer Academic Publishers, 33 AA Dordrecht, Netherlands, 1990.

[9] F. Coudert, B. Richard IRIS 320 GEOV: a new track geometry recording system designed continuing on from the Mauzin track recording coaches, Revue generale des chemins de fer, June, pp. 7-22, 2009.

[10] G. Kerschen, K. Worden, A. F. Vakakis, J.-C. Golinval, Past, present and future of nonlinear system identification in structural dynamics, Mechanical Systems and Signal Processing 20, p.505-592, 2006.

[11] S. Kraft, Parameter identification for a TGV model, PhD thesis, Ecole Centrale Paris, 2012.

[12] M. Peeters, G. Kerschen, J.C. Golinval, Dynamic testing of nonlinear vibrating structures using nonlinear normal modes, Journal of Sound and Vibration, Volume 330, Issue 3, p.486-509, 2011.

[13] G. Kerschen, M. Peeters, J.C. Golinval, A.F. Vakakis, Nonlinear normal modes, Part I: A useful framework for the structural dynamicist, Mechanical Systems and Signal Processing 23 , p.170-194, 2009.

[14] M. Peeters, R. Viguie, G. Serandour, G. Kerschen, J.-C. Golinval, Nonlinear normal modes, Part II: Toward a practical computation using numerical continuation techniques Mechanical Systems and Signal Processing, Volume 23, Issue 1, p.195-216, 2009.

[15] E. Walter, L. Pronzato, Identification of Parametric Models from Experimental Data, Springer, 1997.

[16] B. Hu, Korrelationsbasierte Methode zur Parameteridentifikation nichtlinearer mechanischer Systeme, Fortschrittsberichte VDI, 257, in German.

[17] A. Saltelli, S. Tarantola, F. Campolongno, M. Ratto, Sensitivity analysis in practice, Wiley, 2004. 
[18] J. Nocedal, S. J. Wright, Numerical Optimization, Springer Verlag New York, 1999.

[19] C. Geiger, C. Kanzow, Numerische Verfahren zur Losung unrestringierter Optimierungsaufgaben, Springer, 2002, in German.

[20] M. Syrjakow, Verfahren zur effizienten Parameteroptimierung von Simulationsmodellen, Dissertation, Universitat Karlsruhe, 1997, in German.

[21] Z. Michalewicz, Genetic Algorithms + Data Structures = Evolution Programs, Springer-Verlag Berlin Heidelberg, 1996 